\title{
The relationships between three-axis accelerometer measures of physical activity and motor symptoms in patients with Parkinson's disease: a single-center pilot study
}

Hiroto Ito ${ }^{1 *}$ (D), Daichi Yokoi ${ }^{1,2}$, Rei Kobayashi ${ }^{1}$, Hisashi Okada ${ }^{1}$, Yasukazu Kajita ${ }^{1,3}$ and Satoshi Okuda ${ }^{1}$

\begin{abstract}
Background: Various wearable devices for objectively evaluating motor symptoms of patients with Parkinson's disease (PD) have been developed. Importantly, previous studies have suggested protective effects of physical activity in PD. However, the relationships between conventional clinical ratings for PD and three-axis accelerometer measures of physical activity (e.g., daily physical activity levels [PAL] or metabolic equivalents of task [METs]) are still unclear, particularly for METs. In the current study, we sought to elucidate these relationships on a daily basis, and to clarify optimal predictors for clinical states on a 30-min basis.
\end{abstract}

Methods: Patients who were hospitalized for adjustment of drugs or deep brain stimulation were enrolled. Using waist-worn three-axis accelerometers, PAL and METs parameter data were obtained and compared with UPDRS$3[\mathrm{On}]$ and symptom diary data. We extracted data from the patients' best and worst days, defined by the best and worst UPDRS-3[On] scores, respectively. Thus, 22 data sets from 11 patients were extracted. We examined the correlations and produced scatter plots to represent the relationships, then investigated which METs parameters and activity patterns were the best predictors for "On" and "dyskinesia".

Results: The parameter "mean METs value within the 95-92.5 percentile range on a day (95-92.5 percentile value)" exhibited the strongest correlation with conventional daily clinical ratings (Rho: -0.799 for UPDRS-3[On], 0.803 for On hours $[p<0.001]$ ). Scatter plots suggested that PAL tended to have higher values in patients with involuntary movement. However, METs parameters focusing on higher METs seemed to alleviate this tendency. We clarified that "time over 2.0 METs" and "time over 1.5 METs" could be predictors for "On" and "dyskinesia" on a 30-min basis, respectively (AUROC: 0.779 and $0.959,95 \% \mathrm{Cl}: 0.733-0.824$ and $0.918-1.000$ ). The specificity and sensitivity of the optimal activity pattern for "On" were 0.858 and 0.621 .

(Continued on next page)

\footnotetext{
* Correspondence: hiroto.ito.nagoya.uni.med@gmail.com

'Department of Neurology, National Hospital Organization Nagoya Medical Center, 4-1-1 Sannomaru, Naka-ku, Nagoya, Japan

Full list of author information is available at the end of the article
} 
(Continued from previous page)

Conclusions: This study suggested feasible activity patterns and METs parameters for objective evaluation of motor symptoms on a 30-min or daily basis. Three-axis accelerometer measures focusing on higher METs may be appropriate for evaluating physical activity. Further larger-scale studies are necessary to clarify the validity, reliability, and clinical utility of these objective measures.

Keywords: Parkinson's disease, Physical activity (PAL), Metabolic equivalents of task (METs), Three-axis accelerometer, UPDRS-3, Symptom diary, Wearable device

\section{Background}

Parkinson's disease (PD) is one of the most common age-related neurodegenerative disorders, characterized by various motor symptoms including slowness of movement (bradykinesia) as a main symptom, tremor (particularly at rest), and rigidity [1]. Although some PD symptoms are alleviated by medication and deep brain stimulation (DBS) [2], the effects gradually wear off as the disease progresses [1]. Motor symptoms become more complicated with fluctuation of symptoms (On-Off phenomena) and involuntary movement (dyskinesia) due to long-term use of levodopa [1]. Evaluation of various motor symptoms that gradually progress over time and change dynamically during the day is necessary in management of PD patients.

The current gold standard for evaluation of PD motor symptoms involves subjective evaluation for a certain period with a symptom diary $[3,4]$ and objective rating via doctor's examination at specific time points in clinical settings (e.g., Movement Disorder Society Unified Parkinson Disease Rating Scale [UPDRS]-3) [5]. However, these subjective and objective methods have limitations related to low accuracy and poor temporal resolution, respectively $[4,6]$.

New methods and technologies using accelerometers or gyroscopes for the objective assessment of motor symptoms through different computational methods have been developed to overcome these limitations [6]. Previous assessments of motor symptoms in PD (such as bradykinesia or dyskinesia) have been attempted with systems such as the Parkinson's KinetiGraph ${ }^{\odot}$ system (PKG) [7] or Kinesia ${ }^{\text {тм }}$ [8]. In some studies, however, contradictory results between UPDRS-3 (significant improvement) and measures of a wearable device (no significant improvement) have been reported $[9,10]$. Although unobtrusive devices for patients are generally desirable for better compliance, some studies investigating the relationships between the gold standard and original scores have required $\mathrm{PD}$ patients to operate smartphones $[11,12]$. These recently developed techniques have not yet become part of routine clinical assessment [6], and have not considered using the intensity of physical activity for objective assessment of motor symptoms.
In recent years, evidence suggesting the protective effects of physical activity against PD has accumulated [13]. A meta-analysis reported that the risk of developing PD was reduced in patients with higher daily physical activity levels (PAL) [14]. Animal experiments using PD model mice reported that exercise has protective effects against PD progression [15]. In chronic obstructive pulmonary disease (COPD), PAL reductions measured with a three-axis accelerometer were reported to be the greatest risk factor for death [16]. Metabolic equivalents of task (METs) are also considered to be a useful indicator of physical activity. One MET is the energy expended while sitting at rest. The METs for activity and exercise are calculated as a relative value to rest. Although the importance of maintaining PAL or METs measured with a three-axis accelerometer has been recognized in COPD $[17,18]$, few studies with wearable devices have focused on PAL $[19,20]$ or METs [21] in PD patients. Even in these studies, the potential influence of involuntary movement was not considered [19-21].

Thus, despite the potential utility of evaluating motor symptoms using three-axis accelerometer measures of physical activity and examining these measures in PD patients, their relationships with the conditions of motor symptoms are currently unclear, particularly for METs. Therefore, the current observational study sought to clarify the relationships on a daily basis between conventional clinical ratings (UPDRS-3 [On], On hours in symptom diary) and three-axis accelerometer measures (PAL and METs parameters [described in detail in Methods]) in various conditions among a variety of PD patients. Furthermore, we also investigated which METs parameters and activity patterns were the best predictors for the "On" and "dyskinesia" states on a 30-min basis.

\section{Methods \\ Study population and design}

Thirteen patients who were hospitalized for adjustment of drugs or DBS (patients were thought to exhibit variation in condition during hospitalization) were recruited from the Neurology Department at Nagoya Medical Center, Japan, between June 2017 and March 2018. Eligible participants had a diagnosis of idiopathic Parkinson's disease. Other inclusion criteria were as follows: a) 
absence of severe cognitive impairment (Mini-Mental State Examination scores $>20$ ); b) written consent was granted by the patients' free will. Patients who met any of the following criteria were not included in the study: a) heart failure, COPD or acute diseases such as infectious disease that restrict exercise; b) the research director or research co-workers determined that participation in this study was not appropriate (such as a history of problematic behavior during hospitalization). We did not apply any cut-off points regarding physical activity or severity of PD to patients. Our study was conducted in a hospitalized setting to confirm patients' conditions in more detail and in the same environment. The study protocol was approved by the ethical committee at Nagoya Medical Center. Written informed consent was obtained from all participants in accordance with the declaration of Helsinki.

\section{Objectives}

Our primary objectives were as follows:

- Study I: To screen three-axis accelerometer measures (PAL and METs parameters [described in Data analysis]) to identify relationships (correlations, scatter plots) with conventional clinical ratings (UPDRS-3 [On] and On hours in symptom diary) on a daily basis.

We also investigated which three-axis accelerometer measures (among measures showing stronger correlations in study I) exhibited the largest change between the best and worst days.

- Study II: To investigate which specific METs parameters (described in Data analysis) are the best predictors of the "On" and "dyskinesia" clinical states on a 30-min basis.

In addition, we conducted activity pattern analysis to investigate the specificity and sensitivity of the defined activity patterns and their combinations (described in Data analysis) for "On".

\section{Wearable device}

A commercial three-axis accelerometer (Active Style Pro HJA 750C; OMRON, Kyoto, Japan $[40 \times 52 \times 12 \mathrm{~mm}$, $23.0 \mathrm{~g}])$ was used for assessment of PAL and METs parameters [22]. The monitor collected three-axis acceleration data at $32 \mathrm{~Hz}$, and METs data were recorded in 10 -s epochs. The device was worn on the waist, and continuously monitored patients for more than $10 \mathrm{~h}$ per day. We referred to previous studies regarding this daily duration of assessment [23, 24]. Medical staff checked regularly to confirm the measurement period. The wearing time started between 07:00 a.m. and 07:30 a.m., and finished between 05:30 p.m. and 07:00 p.m. If the installation of the device was not completed, data were excluded. Data outside the wearing time (00:00 a.m. - 07: 00 a.m., 07:00 p.m.- 00:00 p.m.) were also excluded. The time interval between monitoring ranged from 1 to 7 day(s) in DBS patients, and 7 days in drug adjustment patients.

\section{Conventional clinical ratings (UPDRS-3, on hours in symptom diary)}

UPDRS-3 [On] [5] was evaluated 1-2 h after L-dopa administration [On]. In the symptom log, patients recorded On time (easy to move subjectively), intermediate time between On and Off, Off time (not easy to move subjectively), and the presence or absence of subjectively identified dyskinesia. We used the symptom diary data from 07:00 a.m. - 07:00 p.m. in study I, and the data from 07:30 a.m. - 05:30 p.m. in study II. Patients with dyskinesia were defined by the presence of dyskinesia clinically judged by the examiners. Patients with resting tremor were defined according to the sub-score of resting tremor in UPDRS-3 (sub-score $\geq 2$ ).

\section{Approach}

Because our protocol required hospitalization, we did not include healthy controls. Each data set (conventional clinical ratings and 3-axis accelerometer measures) was obtained on a daily basis. In drug adjustment patients, 2-3 opportunities (days) of evaluation were conducted on a daily basis before and after drug adjustment, and in DBS patients, 2-5 opportunities (days) on a daily basis were conducted for each setting of DBS. To reflect various conditions of patients and ensure the same number of data sets ( 2 days) in all patients, we extracted and analyzed data sets from the patients' best and worst days, defined by UPDRS-3 [On] scores. Evaluation using conventional clinical ratings and three-axis accelerometer measures were also performed on the same days. Evaluation was stopped when the patient requested discontinuation, and data from those days were not included. Patients underwent rehabilitation as usual.

\section{Data analysis}

METs determined by this three-axial accelerometer have been reported to be closely correlated with METs calculated using energy expenditure measured by indirect calorimetry $[25,26]$. Basal metabolic rate (BMR) was estimated from a multiple regression equation including age, sex, height and ideal body weight as variables [27]. Total energy expenditure (TEE) was calculated by a manufactured regression equation using METs assessed by the triaxial accelerometer [26]. PAL was calculated by dividing TEE by BMR [28]. 


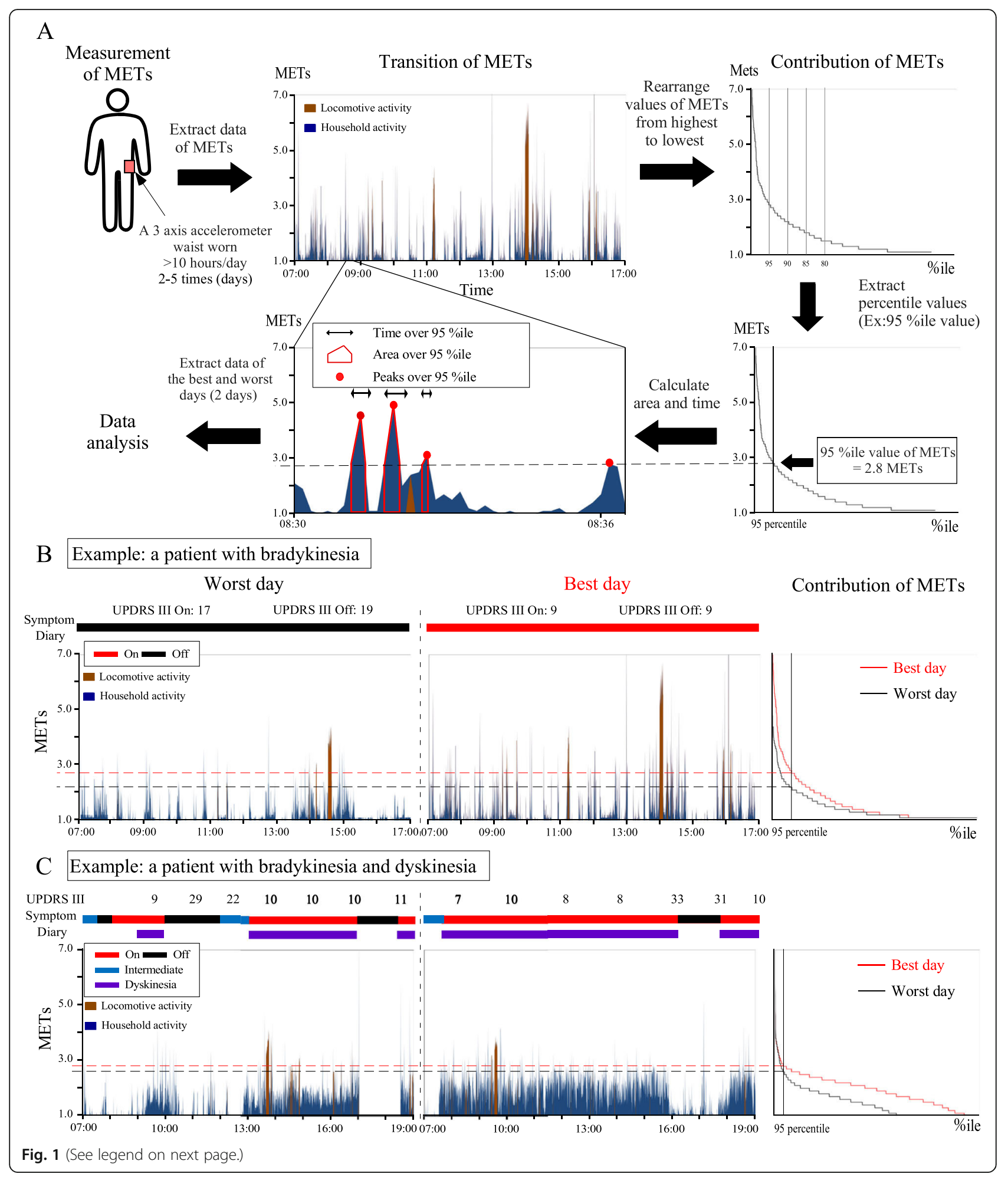


(See figure on previous page.)

Fig. 1 Data processing description and example results. a Data processing flow from measurement to analysis. Patients wore a three-axis accelerometer on the waist for more than $10 \mathrm{~h}$ per day and METs were measured every $10 \mathrm{~s}$. METs data were extracted and lined up over time (transition of METs). METs data were then arranged from highest to lowest values (contribution of METs). Next, percentile values on a day were extracted (in this example case, the 95th percentile value was $2.8 \mathrm{METs}$ ). Finally, time, area and number of maximal values (peaks) over specific percentile values (in this example case, the 95th percentile value $=2.8 \mathrm{METs}$ ) and specific absolute METs values (e.g., 2 METs) were calculated. $\mathbf{b}$ Example results in a patient who had bradykinesia without involuntary movement (transition and contribution of METs, and 95 percentile value) are shown for the days on which the worst or best UPDRS-3 [On] ratings were observed. Transitions of METs appeared to be higher, longer, and more frequent on the patient's best day compared with the patient's worst day. c Example results of a patient with bradykinesia and involuntary movement (dyskinesia), suggesting a correlation between transition of METs with symptom diary states ("On" and "Off") and the elevated baseline of METs (1.5-2.0 METs) during dyskinesia. The patient's condition was checked every 1-2 h(s) objectively using UPDRS-3

The processing flow of the obtained METs data is described in Fig. 1a. METs data were extracted and lined up over time (Transition of METs). METs data were arranged from highest to lowest values (Contribution of METs). Then, each percentile METs value on a day against $12 \mathrm{~h}$ (the wearing time; 4320 epochs [1 epoch/10 s]) was extracted. Finally, the area/time/number of maximal values (peaks) over $(\geq$ ) each percentile value (defined as the mean percentile value on the best and worst days in each patient) and specific METs values were calculated.

In study I, as METs parameters, each mean value within a range of 2.5 percentiles (from the 100th to 70 th percentile) was calculated. Likewise, the area/time/peaks over specific METs values (every 0.5 METs from 1.5 to 3.0 METs) and percentile values (every 2.5 percentiles between the 100th and 90th percentile) were examined. In study II, area/time/peaks over specific METs value (every 0.5 METs from 1.5 to $2.5 \mathrm{METs}$ ) and percentile values (every 2.5 percentiles from the 100th to 90th percentile) on a 30 -min basis were examined.

In the activity pattern analysis, the process of developing the definitions of criteria are described in Supplementary Fig. 3A-C. We defined criteria for activity patterns to distinguish "On" from "Non-On" as follows;

- Criterion I: at least one peak greater than 2.5 METs with four or more consecutive epochs over 2 METs.

- Criterion II: four or more peaks greater than 2.5 METs.

Continuous locomotive activity for more than $1 \mathrm{~min}$ is counted as just one peak.

- Criterion for "tremor": "tremor" was determined by a combination of 90 epochs or more over 1.5 METs and clinical information of resting tremor (sub-score of UPDRS-3 2 2). Tremor is included as "Non-On".

- Exclusion criterion: we excluded peaks over 4 METs.

\section{Statistical analysis}

As this pilot study was exploratory in nature, although no formal sample size estimation was performed, a sample of 11 participants was anticipated to be appropriate for a pilot study.

In study I, Spearman's rank-order correlations were calculated between objective evaluation items measured with the three-axis accelerometer and conventional clinical ratings. We produced scatter plots of the primary results. Changes from the worst to best day of the three-axis accelerometer measures showing stronger correlations in study I were compared using $Z$-scores and nonparametric Wilcoxon signed-rank tests. In study II, the area under the receiver operating characteristic curve (AUROC) was used to assess model performance. In the analysis of "dyskinesia" in study II, the data from the only day on which the patient showed subjective dyskinesia were used in one patient. Statistical analysis was conducted using easy $\mathrm{R}$ version 1.40 . The significance level was set to $p<$ 0.05 .

\section{Results}

Eleven patients (mean age: 67.1 [56-81] years) completed repeated assessments more than twice during hospitalization. Ten patients provided data in symptom diaries (mean recorded time: 11.7 [10.0-12.0] hours). UPDRS-3 [On] and On hours were significantly lower and longer, respectively, on the best day compared with the worst day (mean $\pm S D$ : $18.6 \pm 19.6$ and $8.6 \pm 5.1$ [hours], $30.5 \pm 20.0$ and $1.8 \pm 2.6$ [hours], $p<0.01$ and $p=0.013$, Table 1). Other characteristics are shown in Table 1. Representative data (transition and contribution of METs on both the best and worst days) of patients with bradykinesia (Fig. 1b) and with bradykinesia and dyskinesia (Fig. 1c) are shown. In Fig. 1b, transitions of METs appeared to be higher, longer, and more frequent on the patient's best day compared with the patient's worst day. In Fig. 1c, an elevation of baseline METs (1.5-2.0 METs) was observed during dyskinesia.

Relationships between conventional clinical rating versus three-accelerometer measures on a daily basis in study I

Twenty-two data sets were obtained from 11 patients. A stronger (anti) correlation was observed in the parameter "mean METs value within the 95-92.5 percentile range 
Table 1 Clinical features of the included patients $(n=11)$

\begin{tabular}{ll}
\hline Clinical feature: Mean ( \pm SD) or Number (\%) & \\
\hline Age (years) & $67.1( \pm 7.7)$ \\
Mean disease duration (years) & $13.8( \pm 5.8)$ \\
Gender (\%) & \\
Male & $4(36.4 \%)$ \\
Female & $7(63.6 \%)$ \\
Adjustment of Drugs/DBS (\%) & \\
Drugs & $3(27.3 \%)$ \\
DBS & $8(72.7 \%)$ \\
MMSE & $26.9( \pm 2.7)$ \\
UPDRS-3 (On) & \\
Worst day & $30.5( \pm 20.0)$ \\
Best day & $18.6( \pm 19.6)$ \\
On hour(s) & \\
Worst day & $1.8( \pm 2.6)$ \\
Best day & $8.6( \pm 5.1)$ \\
Motor symptom (\%) & \\
Bradykinesia & $11(100 \%)$ \\
Dyskinesia & $3(27.3 \%)$ \\
Resting tremor & $4(36.4 \%)$ \\
Sub-score of UPDRS-3 22 & $2(18.2 \%)$ \\
Postural instability & $11(100 \%)$ \\
Pulsion phenomenon & $2(18.2 \%)$ \\
Sedactive & $3(27.3 \%)$ \\
\hline Inactive & \\
Active & \\
Phyical activity (\%) & $(36.4 \%)$ \\
\hline
\end{tabular}

SD Standard deviation, DBS Deep brain stimulation, MMSE Mini-Mental State Examination, UPDRS Unified Parkinson's Disease Rating Scale. Sedentary: Usually uses a wheelchair. Inactive: Uses a wheelchair at times. Active: No use of a wheelchair on a day (95-92.5 percentile value)" with both UPDRS-3 and On hours (Rho: $-0.799[p<0.0001]$ and $0.803[p<$ $0.0001]$, Fig. 2) and "area over 92.5 percentile value" with On hours (Rho: $-0.841[p<0.0001]$, Fig. 2). All other screening results are summarized in Fig. 2 and Supplementary table 1 . Regarding the parameter 95-92.5 percentile value, we also identified significant (anti-) correlations on both the best and worst day (Rho: $0.797[p<0.01]$ and $0.682[p<0.05]$ with UPDRS-3; 0.716 and $0.710[p<0.05]$ with On hours), but only on the worst day for the parameter "area over 92.5 percentile value" $(0.623[p=0.054]$ and $0.691[p<0.05]$ with On hours).

We then created scatter plots (UPDRS-3 vs PAL, 9592.5 percentile value, and time over 3.0 METs). Plots of patients exhibiting involuntary movement were located among the higher values of PAL in Fig. 3a. However, by focusing on 95-92.5 percentile value or time over 3.0 METs (considered to be moderate to vigorous physical activity [MVPA] [29]), the tendency for higher values in patients exhibiting involuntary movement appeared to be alleviated (Fig. 3b-c).

Next, we calculated the changes between the worst and best day in the measures showing stronger correlations in study I. Among them, the changes were largest in "area over 92.5 percentile value" (change of Z-score: 1.30, Supplementary Fig. 1).

Three-accelerometer measures as predictors for "on" and "dyskinesia" clinical states on a 30-min basis in study II

The screening results for "On" and "dyskinesia" are summarized in Table 2 and Table 3, and Supplementary table 2A-B. Data were obtained from 10 patients (all patients with bradykinesia, two patients with resting tremor, and two patients with dyskinesia), with 392 and 398 segments (30-min basis) for the analysis of "On" and "dyskinesia". The highest AUROC values for detection of "On" and "dyskinesia" were obtained in "time over 2

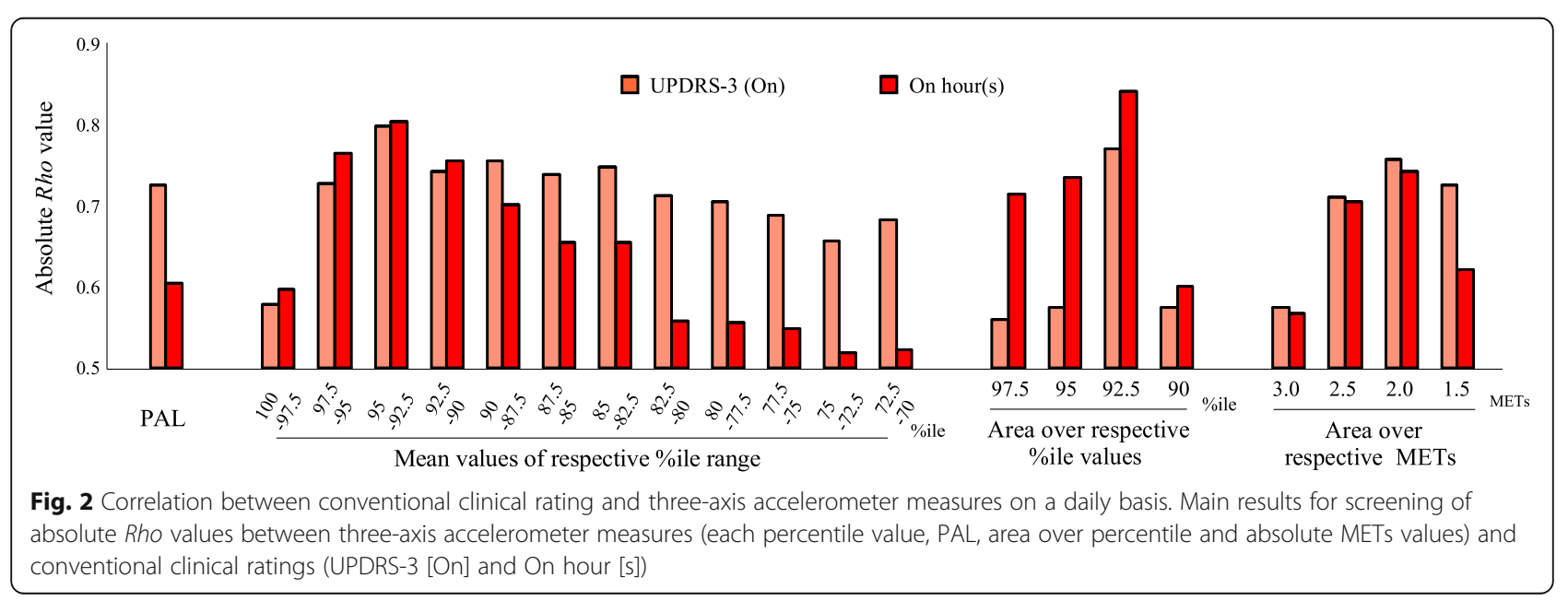




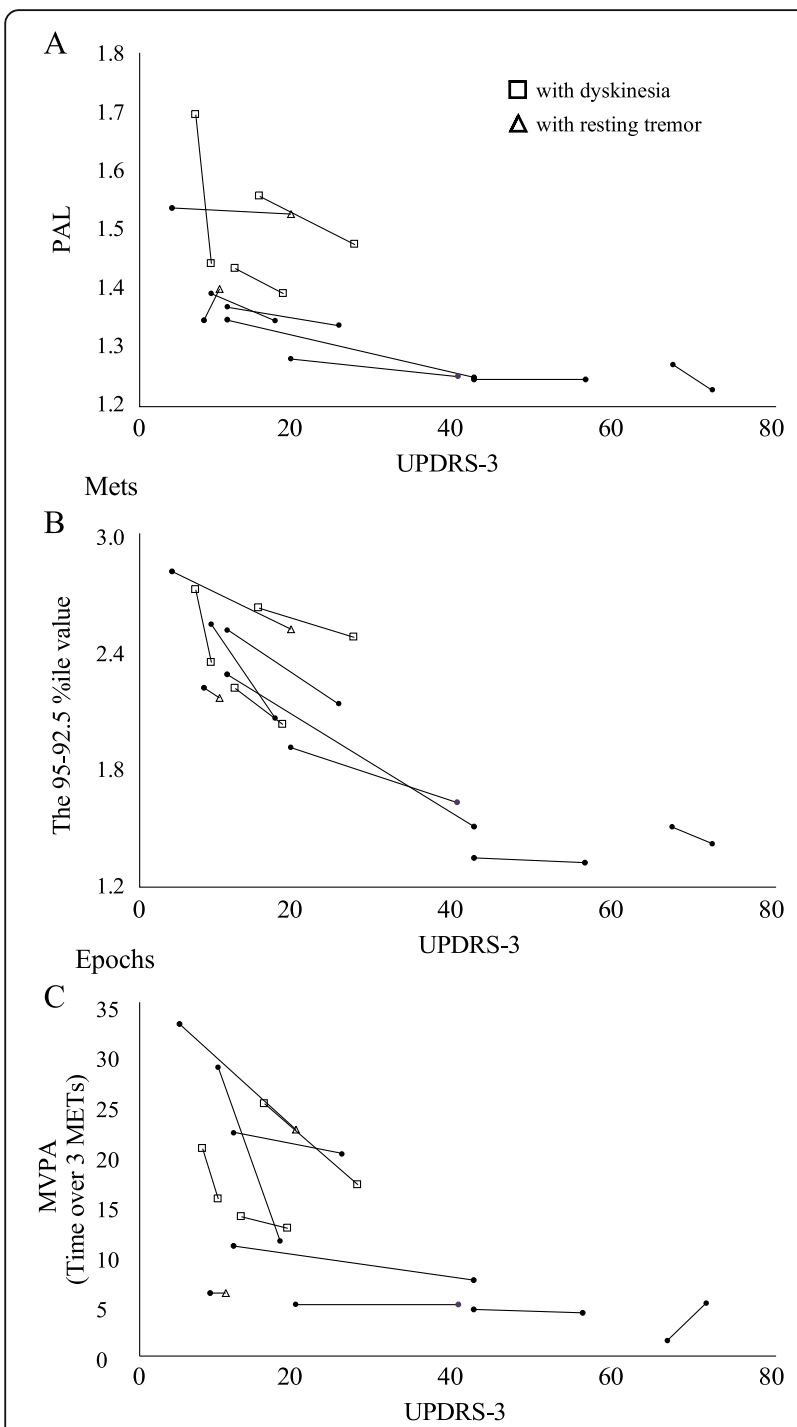

Fig. 3 Scatter plots between conventional clinical rating and threeaxis accelerometer measures on a daily basis. a-c Scatter plots for UPDRS-3 [On] and three-axis accelerometer measures (PAL, "mean METs value within the 95-92.5 percentile range on a day [95-92.5 percentile value]", and "time over 3 METs [moderate to vigorous physical activity; MVPA]"). Plots of each patient's worst and best days were connected with lines. a Plots of patients with involuntary movement ( $\square$ or $\Delta$ ) were located among the higher values of PAL. The triangle $(\Delta)$ shows the day on which the patients had a subscore of resting tremor in UPDRS-3 (sub-score $\geq 2$ ). $\mathbf{b}$-c By focusing on 95-92.5 percentile values or MVPA, the tendency for higher values in patients exhibiting involuntary movement appeared to be alleviated

METs" and "time over 1.5 METs", respectively (AUROC: 0.779 and 0.959 , 95\% CI: $0.733-0.824$ and $0.918-1.000$ ). The specificity and sensitivity of cut-off values (10 and 114 epochs) for these measures, determined by the smallest distance to the top-left corner of the ROC box, were obtained (0.639, and 0.770 for "On" and 0.960 and 0.912 for "dyskinesia"). The application of these cut-off values to each patient is shown in Supplementary Fig. 2A-B.

Next, based on the results of study II, we developed criteria for activity patterns. The process of developing activity pattern criteria to distinguish "On" from "NonOn" is described in Supplementary Fig. 3A-C and their legends. The specificity and sensitivity of these defined activity patterns and their combinations are shown in Supplementary table 3 (0.858 and 0.621 in the optimal combination [criterion I or II, exclusion criterion and criterion for "tremor"]).

\section{Discussion}

From study I, relatively high percentile values (e.g., 9592.5 percentile value) may provide an objective severity measure for PD on a daily basis. The highest percentile values (e.g., 100-97.5 percentile range) were not strongly correlated with conventional ratings. This may be because some patients tended to have more peaks over relatively high METs values ( $\geq 4$ METs), even on their worst day (Supplementary Fig. 3B), possibly due to loss of balance, or pulsion phenomenon. However, these values may not reflect the severity of PD. The lower percentile values (such as $85-70$ percentile range) were not strongly correlated. This may be because lower METs increased in patients exhibiting involuntary movement compared with patients exhibiting only bradykinesia (Supplementary Fig. 2B).

Our results also suggest that involuntary movements in PD patients may increase PAL. This may be due to the elevated baseline of METs. Although several previous studies have investigated PAL using wearable devices, previous studies did not consider the influence of involuntary movement $[19,20]$. The current results suggest that direct application of PAL measured with a three-axis accelerometer is not suitable for evaluating physical activity. Duration of physical activity over higher METs values (e.g., MVPA [29]) may be appropriate for evaluating physical activity in PD patients .

In the activity pattern analysis, we proposed activity patterns corresponding to "On", "Non-On", "tremor" and "dyskinesia" (Supplementary Fig. 3B). Higher, longer or more frequent increases in METs values may be explained by improvement of PD symptoms (slowness and less frequency of voluntary movement [1]), and lead to high performance of "95-92.5 percentile value" and "time over 2 METs". The elevated baseline of METs in involuntary movement, "dyskinesia" was in accord with the high performance of "time over 1.5 METs".

To the best of our knowledge, the current study is the first to use a commercially available three-axis accelerometer to investigate the relationships between conventional clinical ratings and METs parameters. The requirements for patients were simple; wearing the 
Table 2 Compariosn among METs parameters in predection for "On"

\begin{tabular}{llll}
\hline Parameters & AUROC & $95 \% \mathrm{Cl}$ & P-value \\
\hline Area & & & \\
Over 1.5 METs & 0.747 & $0.698-0.795$ & $<0.001$ \\
Over 2 METs & 0.773 & $0.727-0.819$ & 0.030 \\
Over 2.5 METs & 0.752 & $0.705-0.800$ & 0.033 \\
Over 3.0 METs & 0.673 & $0.621-0.725$ & $<0.001$ \\
Time & & & \\
Over 1.5 METs & 0.740 & $0.692-0.789$ & $<0.001$ \\
Over 2 METs & 0.779 & $0.733-0.824$ & reference \\
Over 2.5 METs & 0.766 & $0.719-0.813$ & 0.24 \\
Over 3.0 METs & 0.671 & $0.619-0.723$ & $<0.001$ \\
Peaks & & & $<0.01$ \\
Over 1.5 METs & 0.744 & $0.695-0.792$ & 0.026 \\
Over 2 METs & 0.765 & $0.719-0.812$ & 0.41 \\
Over 2.5 METs & 0.770 & $0.723-0.816$ & $<0.001$ \\
Over 3.0 METs & 0.686 & $0.635-0.737$ & \\
\hline
\end{tabular}

Table 3 Compariosn among METs parameters in predection for "dyskinesia"

\begin{tabular}{llll}
\hline Parameters & AUROC & $95 \% \mathrm{Cl}$ & $P$-value \\
\hline Area & & & \\
Over 1.5 METs & 0.956 & $0.913-0.999$ & 0.21 \\
Over 2 METs & 0.932 & $0.884-0.981$ & 0.011 \\
Over 2.5 METs & 0.859 & $0.785-0.933$ & $<0.001$ \\
Over 3.0 METs & 0.677 & $0.598-0.756$ & $<0.001$ \\
Time & & & \\
Over 1.5 METs & 0.959 & $0.918-1.000$ & reference \\
Over 2 METs & 0.943 & $0.897-0.989$ & 0.062 \\
Over 2.5 METs & 0.872 & $0.799-0.946$ & $<0.01$ \\
Over 3.0 METs & 0.678 & $0.595-0.760$ & $<0.001$ \\
Peaks & & & 0.16 \\
Over 1.5 METs & 0.942 & $0.881-1.000$ & 0.041 \\
Over 2 METs & 0.930 & $0.862-0.998$ & 0.010 \\
Over 2.5 METs & 0.870 & $0.782-0.957$ & $<0.001$ \\
Over 3.0 METs & 0.713 & $0.622-0.804$ & \\
\hline
\end{tabular}

AUROC Area under receiver operating curve, $\mathrm{Cl}$ Confidence interval; $M E T$, metabolic equivalents of task. (A) We compared the performance of METs parameters as predictive models for the "On" state, with estimates of the area under the receiver operating characteristic curve (AUROC). The performance of time over 2 METs (AUROC: $0.779,95 \% \mathrm{Cl}: 0.733-0.824$ ) was higher than that of time and peaks over 2.5 METs and was significantly higher than the others. (B) We compared the performance of METs parameters as predictive models for the "dyskinesia" state, with estimates of AUROC. The performance of time over 1.5 METs (AUROC: $0.959,95 \% \mathrm{Cl}: 0.918-1.000$ ) was higer than that of area and peaks over 1.5 METs and time over 2 METs, and significantly higher than the others device only on the waist. This compact device may be feasible for clinical applications. For instance, it may be possible to objectively rate the gradual progression of disease severity by "95-92.5 percentile value" or the state on a 30-min basis by the defined activity pattern.

One limitation was the lack of a thorough evaluation in a daily life environment which is necessary to confirm the appropriateness of the device for outpatients. As for other limitations, we did not monitor the patients during the night period and for a more extended period of time (e.g., more than 3 days). Moreover, possible artefacts were not excluded, since we could not deny the possibility that higher METs might be caused by some activities, such as loss of balance or pulsion phenomenon. The results should be interpreted with caution because of the small sample size (particularly, a small number of patients with dyskinesia), and the absence of a healthy control group. Since we did not collect "tremor" state in the symptom diary, it is currently unclear whether dyskinesia is distinguishable from tremor based on only the data from our device.

\section{Conclusions}

In study I, 95-92.5 percentile value exhibited the strongest correlation with conventional daily clinical ratings. Scatter plots suggested that objective measures focusing on higher METs (e.g., MVPA) may be appropriate for evaluating physical activity. In study II, the optimal predictors for "On" and "dyskinesia" were "time over 2.0 METs" and "time over 1.5 METs", respectively. The defined activity patterns may be feasible predictors for "On". Further larger-scale studies are necessary to clarify the validity, reliability, and clinical utility of these objective measures.

\section{Supplementary information}

Supplementary information accompanies this paper at https://doi.org/10. 1186/s12883-020-01896-w.

Additional file 1: Figure S1. Changes between worst and best day of three-axis accelerometer measures. $+; p<0.01, \neq ; p<0.001$. Every measure was standardized using Z-scores, then compared. In the three axis accelerometer measures showing stronger correlations in study I, the largest changes were observed in "area over 92.5 percentile value (defined as the mean 92.5 percentile value on the best and worst days in each patient)". The mean $Z$ scores on the best and worst day are connected with a red line.

Additional file 2: Figure S2. Dot-plots of "time over 1.5 and 2 METs" and application of their cut-off values. Int, intermediate; Pt, patient. (A) Data were obtained from 10 patients, with 392 segments (dots). As a candidate for a cut-off value of "time over 2.0 METs", 10 (epochs) were considered (red dashed line). (B) Data were obtained from 10 patients (six patients with bradykinesia, two patients with resting tremor, and two patients with dyskinesia), with 398 segments (dots). We considered 114 (epochs) as a candidate for the cut-off value of "time over 1.5 METs" (blue dashed line). Supplementary fig. 2B showed that patients with resting tremor also had values over the cut-off values. The application of the cut- 
off value for patient 11 (a patient with dyskinesia, who could not record a symptom diary) suggests increased dyskinesia time on the best day.

Additional file 3: Figure S3. Production process and criteria of activity patterns to distinguish "On" from "Non-On". (A) ROC curve of "time over 2 METs" and "peaks over 2.5 METs". To develop the criteria, we focused on the better predictors "time over 2 METs" and "peaks over 2.5 METs" for "On" (Tables 2 and 3). The cut-off values of these measures determined by the smallest distance to the top-left corner of the ROC box were 10 and 3 epochs, respectively. From these cut-off values, we calculated four ( $>10$ epochs/3 epochs $=3.3$ ) or more consecutive epochs over 2 METs in Criterion I and four or more peaks greater than $2.5 \mathrm{MET}$ in Criterion II. (B) The percentage and number of peaks over 4 METs on the worst and best days. The percentage appeared to be low (mean percentage; 3.1 [1.2-6.2] and $2.0[0.81-5.3], p=0.12$; mean number; 13.7 [7-28] and 12.6 [8-34], $p=$ 0.51 ), and a relatively higher percentage and larger number were observed on the worst day, suggesting that some PD patients on the worst day tended to have more peaks over 4 METs. On the basis of these results, we developed the criteria for exclusion of peaks $\geq 4$ METs. (C) Criteria for activity patterns to distinguish "On" from "Non-On". The definitions of the criteria for "On", "Non-On" and "tremor (resting tremor)" . The criteria for dyskinesia are also described for reference. Regarding "tremor" (the sub-score of resting tremor in UPDRS-3 $\geq 2$ ), we focused on "time over 1.5 METs". A cut-off value of 90 epochs was determined, because patients with resting tremor showed milder elevation of baseline of METs compared with dyskinesia (reference) and almost all patients with bradykinesia did not have values over 90 epochs (Supplementary Fig. 1B). "Tremor" was classified as "Non-On", even when patients met the criteria for "On".

Additional file 4: Table S1. All screening results in study I.

Additional file 5: Table S2. Comparing state prediction (On, dyskinesia) using three-axis accelerometer measures (percentile) on a 30 -min basis with ROC curve analysis in study II.

Additional file 6: Table S3. Specificity and sensitivity of the defined activity patterns and their combination.

\section{Abbreviations}

PD: Parkinson's disease; DBS: Deep brain stimulation; PAL: Physical activity levels; MET(s): Metabolic equivalent(s) of task; UPDRS-3: Movement Disorder Society Unified Parkinson Disease Rating Scale part 3; MVPA: Moderate to vigorous physical activity; COPD: Chronic obstructive pulmonary disease; BMR: Basal metabolic rate; TEE: Total energy expenditure; AUROC: Area under the receiver operating characteristic curve

\section{Acknowledgments}

The authors are grateful to Sho Nagayoshi (OMRON Healthcare), who kindly supported us in the use of Active Style Pro HJA-750C. We appreciate the help and support of all the members of the Luncheon Meeting for Clinical Research and the staffs of Clinical Research Center in Nagoya Medical Center, particularly Dr. Akane Kunitomi (Otsu Red Cross Hospital), Akiko Kada, MPH. Dr. Toshiki Saito, Dr. Hirokazu Nagai and Dr. Keizo Horibe (Clinical Research Center, Nagoya Medical Center). We are thankful to medical staffs in the department of neurology at Nagoya Medical Center, particularly PT. Satoshi Maruyama, PT. Shinichi Watanabe, PT. Chie Yamada and PT. Yoshitaka Akiyama. We thank Daisy Bugarin, Mickey TerLouw (Nagoya University) and Benjamin Knight, MSc., (Edanz Group) for English language editing.

\section{Authors' contributions}

$\mathrm{HI}, \mathrm{DY}, \mathrm{RK}, \mathrm{HO}, \mathrm{YK}$, and SO contributed to the design of the study and undertook the clinical management. HI, DY and YK contributed to acquisition of data. HI, RK, and SO contributed to analysis and interpretation of data. $\mathrm{HI}$ drafted the first manuscript and reviewed the literature. RK, $\mathrm{HO}$, YK and SO critically revised the manuscript. All authors have read and approved the final manuscript.

\section{Funding}

None of the authors received any financial assistance for this work.

\section{Availability of data and materials}

The datasets used and analyzed during the current study are available from the corresponding author on reasonable request.

\section{Ethics approval and consent to participate}

The study protocol was approved by the Ethical committee (Nagoya Medical Center). Written informed consent was obtained from all participants in accordance with the declaration of Helsinki.

\section{Consent for publication}

Not applicable.

\section{Competing interests}

Declarations of interest: none.

\section{Author details}

${ }^{1}$ Department of Neurology, National Hospital Organization Nagoya Medical Center, 4-1-1 Sannomaru, Naka-ku, Nagoya, Japan. ${ }^{2}$ Department of Neurology, Kakeyu Misayama rehabilitation center, 1308 Kakeyuonsen, Ueda, Nagano, Japan. ${ }^{3}$ Department of Neurosurgery, National Hospital Organization Nagoya Medical Center, 4-1-1 Sannomaru, Naka-ku, Nagoya, Aichi, Japan.

Received: 18 February 2020 Accepted: 19 August 2020

Published online: 10 September 2020

\section{References}

1. Kalia LV, Lang AE. Parkinson's disease. Lancet. 2015;386(9996):896-912. https://doi.org/10.1016/s0140-6736(14)61393-3.

2. Deuschl G, Schade-Brittinger C, Krack P, Volkmann J, Schafer H, Botzel K, et al. A randomized trial of deep-brain stimulation for Parkinson's disease. $\mathrm{N}$ Engl J Med. 2006;355(9):896-908. https://doi.org/10.1056/NEJMoa060281.

3. Montgomery GK, Reynolds NC Jr. Compliance, reliability, and validity of selfmonitoring for physical disturbances of Parkinson's disease. The Parkinson's Symptom Diary. J Nerv Mental Dis. 1990;178:636-41. https://doi.org/10.1097/ 00005053-199010000-00004.

4. Reimer J, Grabowski M, Lindvall O, Hagell P. Use and interpretation of on/off diaries in Parkinson's disease. J Neurol Neurosurg Psychiatry. 2004;75(3):396400. https://doi.org/10.1136/jnnp.2003.022780.

5. Goetz CG, Tilley BC, Shaftman SR, Stebbins GT, Fahn S, Martinez-Martin P, et al. Movement Disorder Society-sponsored revision of the unified Parkinson's disease rating scale (MDS-UPDRS): scale presentation and clinimetric testing results. Mov Disord. 2008;23(15):2129-70. https://doi.org/ 10.1002/mds.22340.

6. Maetzler W, Domingos J, Srulijes K, Ferreira JJ, Bloem BR. Quantitative wearable sensors for objective assessment of Parkinson's disease. Mov Disord. 2013:28(12):1628-37. https://doi.org/10.1002/mds.25628.

7. Griffiths RI, Kotschet K, Arfon S, Xu ZM, Johnson W, Drago J, et al. Automated assessment of bradykinesia and dyskinesia in Parkinson's disease. J Parkinsons Dis. 2012;2(1):47-55. https://doi.org/10.3233/JPD-201211071.

8. Giuffrida JP, Riley DE, Maddux BN, Heldman DA. Clinically deployable Kinesia technology for automated tremor assessment. Mov Disord. 2009;24(5):72330. https://doi.org/10.1002/mds.22445.

9. Johansson D, Ericsson A, Johansson A, Medvedev A, Nyholm D, Ohlsson F, et al. Individualization of levodopa treatment using a microtablet dispenser and ambulatory accelerometry. CNS Neurosci Ther. 2018;24(5):439-47. https://doi.org/10.1111/cns.12807.

10. Isaacson SH, Boroojerdi B, Waln O, McGraw M, Kreitzman DL, Klos K, et al. Effect of using a wearable device on clinical decision-making and motor symptoms in patients with Parkinson's disease starting transdermal rotigotine patch: a pilot study. Parkinsonism Relat Disord. 2019;64:132-7. https://doi.org/10.1016/j.parkreldis.2019.01.025.

11. Zhan A, Mohan S, Tarolli C, Schneider RB, Adams JL, Sharma S, et al. Using smartphones and machine learning to quantify Parkinson disease severity. JAMA Neurology. 2018;75:7. https://doi.org/10.1001/jamaneurol.2018.0809.

12. Lipsmeier F, Taylor Kl, Kilchenmann T, Wolf D, Scotland A, Schjodt-Eriksen J, et al. Evaluation of smartphone-based testing to generate exploratory outcome measures in a phase 1 Parkinson's disease clinical trial. Mov Disord. 2018;33(8):1287-97. https://doi.org/10.1002/mds.27376. 
13. Marras C, Canning CG, Goldman SM. Environment, lifestyle, and Parkinson's disease: implications for prevention in the next decade. Mov Disord. 2019; 34(6):801-11. https://doi.org/10.1002/mds.27720.

14. Yang F, Trolle Lagerros Y, Bellocco R, Adami HO, Fang F, Pedersen NL, et al. Physical activity and risk of Parkinson's disease in the Swedish National March Cohort. Brain. 2015;138(Pt 2):269-75. https://doi.org/10.1093/brain/ awu323.

15. Petzinger GM, Fisher BE, McEwen S, Beeler JA, Walsh JP, Jakowec MW. Exercise-enhanced neuroplasticity targeting motor and cognitive circuitry in Parkinson's disease. Lancet Neurol. 2013;12(7):716-26. https://doi.org/10. 1016/s1474-4422(13)70123-6.

16. Waschki B, Kirsten A, Holz O, Muller KC, Meyer T, Watz H, et al. Physical activity is the strongest predictor of all-cause mortality in patients with COPD: a prospective cohort study. Chest. 2011;140(2):331-42. https://doi. org/10.1378/chest.10-2521.

17. Minakata Y, Sugino A, Kanda M, Ichikawa T, Akamatsu K, Koarai A, et al. Reduced level of physical activity in Japanese patients with chronic obstructive pulmonary disease. Respir Investig. 2014;52(1):41-8. https://doi. org/10.1016/j.resinv.2013.06.002.

18. Minakata Y, Motegi T, Ueki J, Gon Y, Nakamura S, Anzai T, et al. Effect of tiotropium/olodaterol on sedentary and active time in patients with COPD: post hoc analysis of the VESUTO((R)) study. Int J Chron Obstruct Pulmon Dis. 2019;14:1789-801. https://doi.org/10.2147/COPD.S208081.

19. Dontje ML, de Greef MHG, Speelman AD, van Nimwegen M, Krijnen WP, Stolk RP, et al. Quantifying daily physical activity and determinants in sedentary patients with Parkinson's disease. Parkinsonism Relat Disord. 2013; 19(10):878-82. https://doi.org/10.1016/j.parkreldis.2013.05.014.

20. Galperin I, Hillel I, Del Din S, Bekkers EMJ, Nieuwboer A, Abbruzzese G, et al. Associations between daily-living physical activity and laboratory-based assessments of motor severity in patients with falls and Parkinson's disease. Parkinsonism Relat Disord. 2019;62:85-90. https://doi.org/10.1016/j.parkreldis. 2019.01.022.

21. Naoshi SAITOTY, Yoshihiro SUGIURA, Saori SHIMIZU, Masaru SHIMIZU. Lifecorder: a new device for the long-term monitoring of motor activities for Parkinson's disease. Intern Med. 2004;43(8):685-92.

22. Hamasaki H, Yanai H, Kakei M, Noda M, Ezaki O. The association between daily physical activity and plasma B-type natriuretic peptide in patients with glucose intolerance: a cross-sectional study. BMJ Open. 2015;5:e006276. https://doi.org/10.1136/bmjopen-2014-006276.

23. Chen T, Kishimoto H, Honda T, Hata J, Yoshida D, Mukai N, et al. Patterns and levels of sedentary behavior and physical activity in a general Japanese population: the Hisayama study. J Epidemiol. 2018;28(5):260-5. https://doi. org/10.2188/jea.JE20170012.

24. Trost SG, Mclver KL, Pate RR. Conducting accelerometer-based activity assessments in field-based research. Med Sci Sports Exerc. 2005;37(11 Suppl):S531-43. https://doi.org/10.1249/01.mss.0000185657.86065.98.

25. Oshima Y, Kawaguchi K, Tanaka S, Ohkawara K, Hikihara Y, Ishikawa-Takata K, et al. Classifying household and locomotive activities using a triaxial accelerometer. Gait Posture. 2010;31(3):370-4. https://doi.org/10.1016/j. gaitpost.2010.01.005

26. Ohkawara K, Oshima Y, Hikihara Y, Ishikawa-Takata K, Tabata I, Tanaka S. Real-time estimation of daily physical activity intensity by a triaxial accelerometer and a gravity-removal classification algorithm. Br J Nutr. 2011; 105(11):1681-91. https://doi.org/10.1017/s0007114510005441.

27. Ganpule AA, Tanaka S, Ishikawa-Takata K, Tabata I. Interindividual variability in sleeping metabolic rate in Japanese subjects. Eur J Clin Nutr. 2007;61(11): 1256-61. https://doi.org/10.1038/sj.ejcn.1602645.

28. Energy and protein requirements. Report of a joint FAO/WHO/UNU Expert Consultation. World Health Organ Tech Rep Ser. 1985;724:1-206.

29. Cavalheri V, Straker L, Gucciardi DF, Gardiner PA, Hill K. Changing physical activity and sedentary behaviour in people with COPD. Respirology. 2016; 21(3):419-26. https://doi.org/10.1111/resp.12680.

\section{Publisher's Note}

Springer Nature remains neutral with regard to jurisdictional claims in published maps and institutional affiliations.

\section{Ready to submit your research? Choose BMC and benefit from:}

- fast, convenient online submission

- thorough peer review by experienced researchers in your field

- rapid publication on acceptance

- support for research data, including large and complex data types

- gold Open Access which fosters wider collaboration and increased citations

- maximum visibility for your research: over $100 \mathrm{M}$ website views per year

At BMC, research is always in progress.

Learn more biomedcentral.com/submissions 